\title{
Perirenal Perivascular Epithelioid Cell Tumor
} (PEcoma) with Pulmonary Micro Invasive Adenocarcinoma: A Case Report and Literature
Review

Zhaoxun $\mathrm{Li}^{1, *}$

$\mathrm{Nan} \mathrm{Hu}^{2}$,*

Yan Liao'

Jingmei Liu'

'Department of Gastroenterology, Tongji Hospital, Tongji Medical College,

Huazhong University of Science and

Technology, Wuhan, 430030, Hubei,

People's Republic of China; ${ }^{2}$ Department

of Neurology and Laboratory of Clinical

Genetics, Peking Union Medical College

Hospital, Beijing, 100730, People's

Republic of China

*These authors contributed equally to this work
Correspondence: Jingmei Liu

Department of Gastroenterology, Tongji Hospital of Tongii Medical College,

Huazhong University of Science and

Technology, Wuhan, 430030, People's

Republic of China

$\mathrm{Tel} / \mathrm{Fax}+8627836650 \mathrm{II}$

Email LJM890202@I63.com

\begin{abstract}
Perivascular epithelioid cell tumor (PEComa) is a rare type of mesenchymal neoplasm, which occurs most commonly in uterus and gastrointestinal tract. PEComa with perirenal manifestation is an extremely rare entity. To the best of our knowledge, only four cases have been reported up to now. In this case, we reported a patient with both a pulmonary mass and a perirenal mass. Two resections were performed successively and postoperative pathology suggested pulmonary micro invasive adenocarcinoma (MIA) and perirenal PEComa. This is the first case of perirenal PEComa with pulmonary MIA. Combining the present case and prior literature, we summarized the crucial role of immunohistochemistry in the diagnosis and consider that complete operation might be conducive to patients with perirenal PEComa that presents a benign phenotype, regardless of complications with other tumors.
\end{abstract}

Keywords: perivascular epithelioid cell tumor, pulmonary micro invasive adenocarcinoma, case report

\section{Introduction}

Perivascular epithelioid cell tumor (PEComa), first described by Zamboni et al. in 1996, ${ }^{1}$ is a rare type of mesenchymal neoplasm. In 2007, the World Health Organization (WHO) formally defined it as "a kind of mesenchymal tumors composed of histologically and immunohistochemically distinctive perivascular epithelioid cells".2. According to the distinctive morphology and expression of myomelanocytic markers, the PEComa family mainly includes angiomyolipoma (AML), clear cell "sugar" tumor of the lung (CCST), lymphangioleiomyomatosis (LAM), and a number of unusual visceral, intraabdominal, soft tissue and bone tumors. ${ }^{3}$ PEComa most commonly occurs in the uterus (including uterine and cervix uteri) ${ }^{4}$ and gastrointestinal tract. ${ }^{5}$ Perirenal manifestation is an extremely rare entity. To the best of our knowledge, only four cases have been reported up to now, ${ }^{6-9}$ among which only one case study ${ }^{6}$ described a case in which the perirenal PEComa coexisted with malignant gastroenteric tumors. No certain origin and uniform histological pattern has been detected yet. ${ }^{10}$ The treatment of PEComa remains controversial; complete resection is the mainstay of treatment while chemotherapy and immunotherapy are also applied in some cases.

In this study we report the first case of perirenal PEComa with pulmonary micro invasive adenocarcinoma (MIA) ${ }^{11}$ and discuss the diagnosis and treatment of the perirenal PEComa. 


\section{Case Report}

The study was approved by the Ethics Committee of Tongji hospital, Tongji Medical College, Huazhong University of Science and Technology, China. Written informed consent for publication of the details was obtained from the patient.

A 43-year-old man presented to the department of thoracic surgery with a $13 \times 4 \mathrm{~mm}$ nodule in the right upper lung detected by computed tomography (CT) during examination (Figure 1A). This patient had no clinical symptoms and no history of smoking. Tumor marker tests showed that the level of anti-CAGE antibody was slightly elevated $(8.8 \mathrm{U} / \mathrm{mL}$, normally $\leq 7.2 \mathrm{U} / \mathrm{L})$, which is commonly thought to be a lung cancer-associated autoantibody. Furthermore, a perirenal mass, $18 \times 28 \mathrm{~mm}$ in size, close to the hilum of the left kidney was found through abdominal magnetic resonance imaging (MRI) (Figure 1B). Positron emission tomography-computed tomography (PET-CT) showed lesions in both the lung and the renal hilum (Figure 2A and B), which suggested neoplastic lesions. Since the main complaint of the patient was the pulmonary neoplasm, firstly he was scheduled for sublobectomy of right upper lung tip by video-assisted thoracic surgery (VATS). The postoperative pathology indicated that the lung nodule was microinvasive lung adenocarcinoma (Supplementary Figure $1 \mathrm{~A}$ and B).
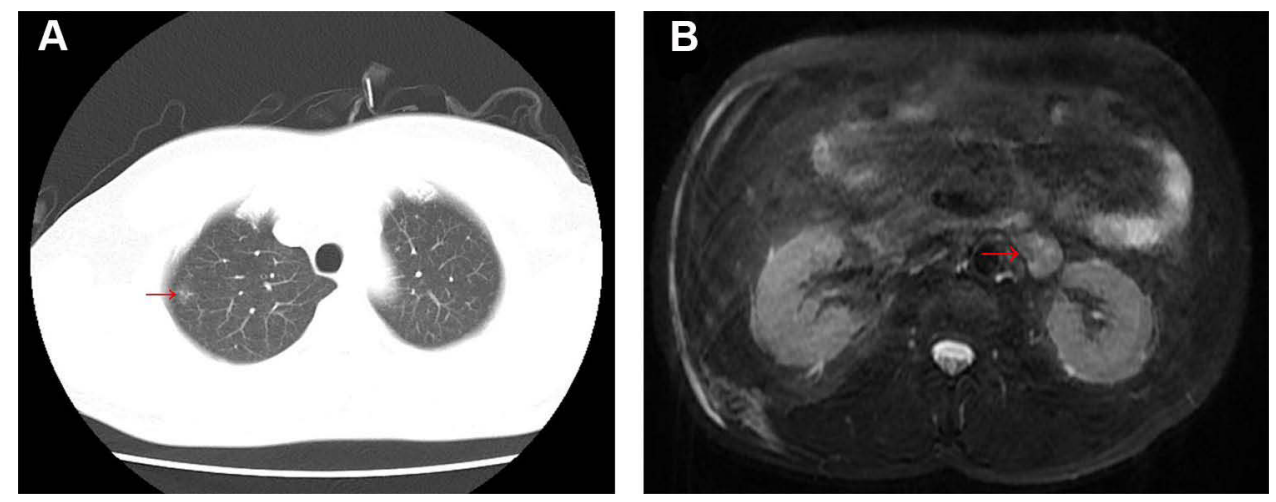

Figure I (A) Chest CT detected a $13 \times 4 \mathrm{~mm}$ nodule in the right upper lung; (B) abdominal MRI revealed a perirenal tumor, I8×28 mm in size, close to the hilum of the left kidney.
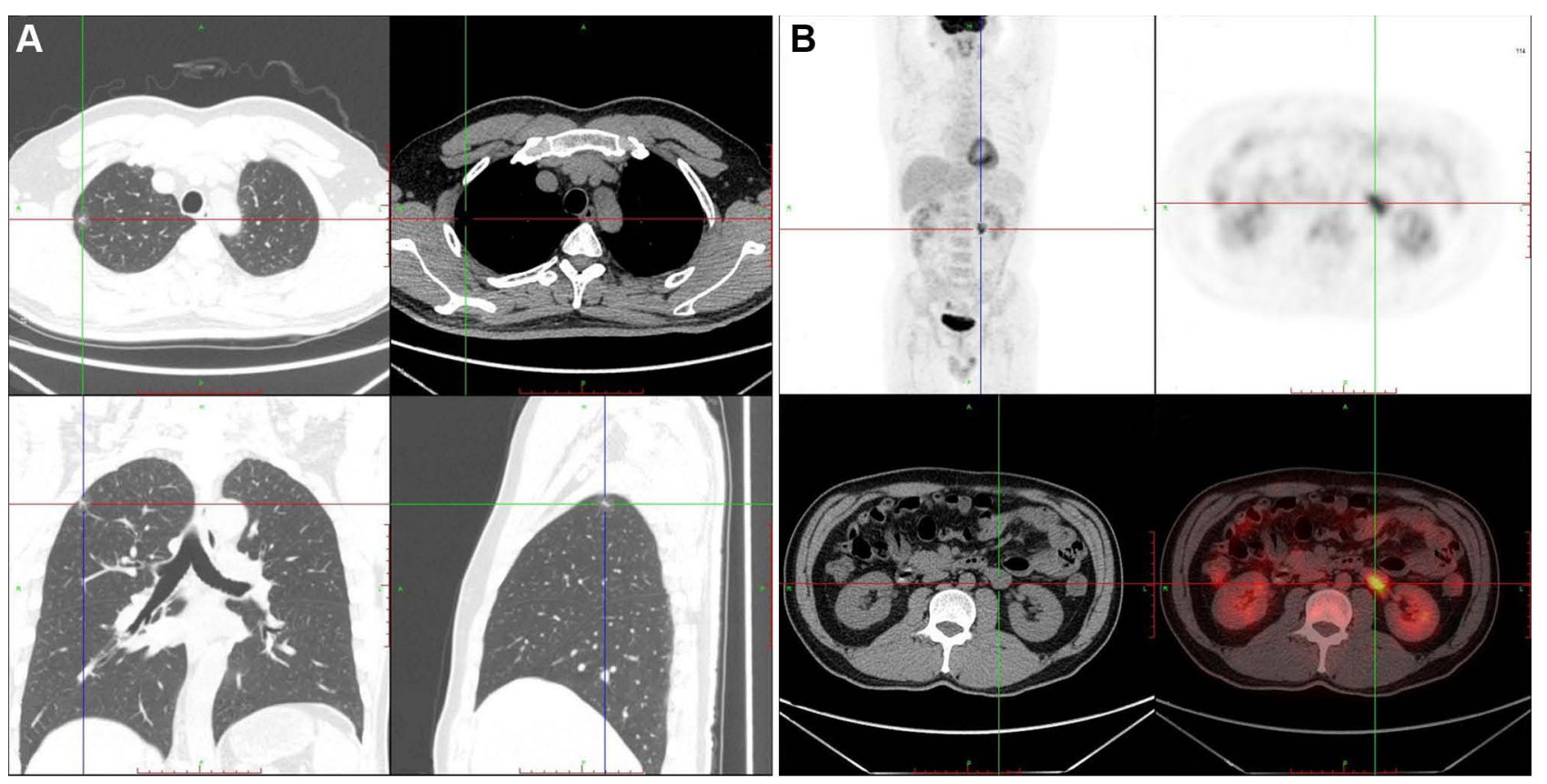

Figure 2 Positron emission tomography (PET)-CT whole body imaging. (A) A $15 \times 10 \mathrm{~mm}$ shadow with ground glass density in the upper right lung was detected; (B) a neoplastic lesion with hypermetabolism close to the left renal hilum was shown. 
Furthermore, immunohistochemical stains showed that this lesion was C-MET $(+)$ and ROS1 (+) (Supplementary Figure $1 \mathrm{C}$ and D). Considering the patient's physical condition, he went home for two months after the first operation and was then admitted to our hospital for retroperitoneal neoplasm. Abdominal enhanced CT suggested a $22 \times 30 \mathrm{~mm}$ mass inferior to the left renal vein with enhancement but no sign of local and vascular invasion. Hormone tests showed normal levels of renin, aldosterone, cortisol and epinephrine. To further define the nature of the mass, endoscopic ultrasound-guided fine needle aspiration (EUS-FNA) was performed and the histopathology of the biopsy showed tumor cells. Subsequently, laparotomy was conducted and the postoperative period was uneventful. Macroscopically, the perirenal tumor consisted of a grayish yellow fragile tissue fragment measuring $40 \times 20 \times 10 \mathrm{~mm}$. Histologically, the tumor was composed of cells with eosinophilic cytoplasm with moderate epithelioid appearance (Supplementary Figure 2A-D). No prominent nuclear polymorphism was shown in most cells. Further immunohistochemical stains were performed on the perirenal lesion and showed that the lesion was positive for Melan-A, cluster differentiation (CD)56 and TFE3, while negative for HMB45, CgA, Syn, S-100 and SOX10. In addition, Ki67 was present in approximately $1 \%$ of tumor cells (Figure 3 ). The histological examination and immunohistochemical stains fulfilled the criteria for the diagnosis of benign PEComa. Finally, the patient was identified as a benign perirenal PEComa coexisting with micro invasive lung adenocarcinoma (T1aN0M0R0). The patient was discharged on the 10th postoperative day.

\section{Discussion}

Since the first case of PEComa reported in 1996, cases involving various anatomic locations have been reported,

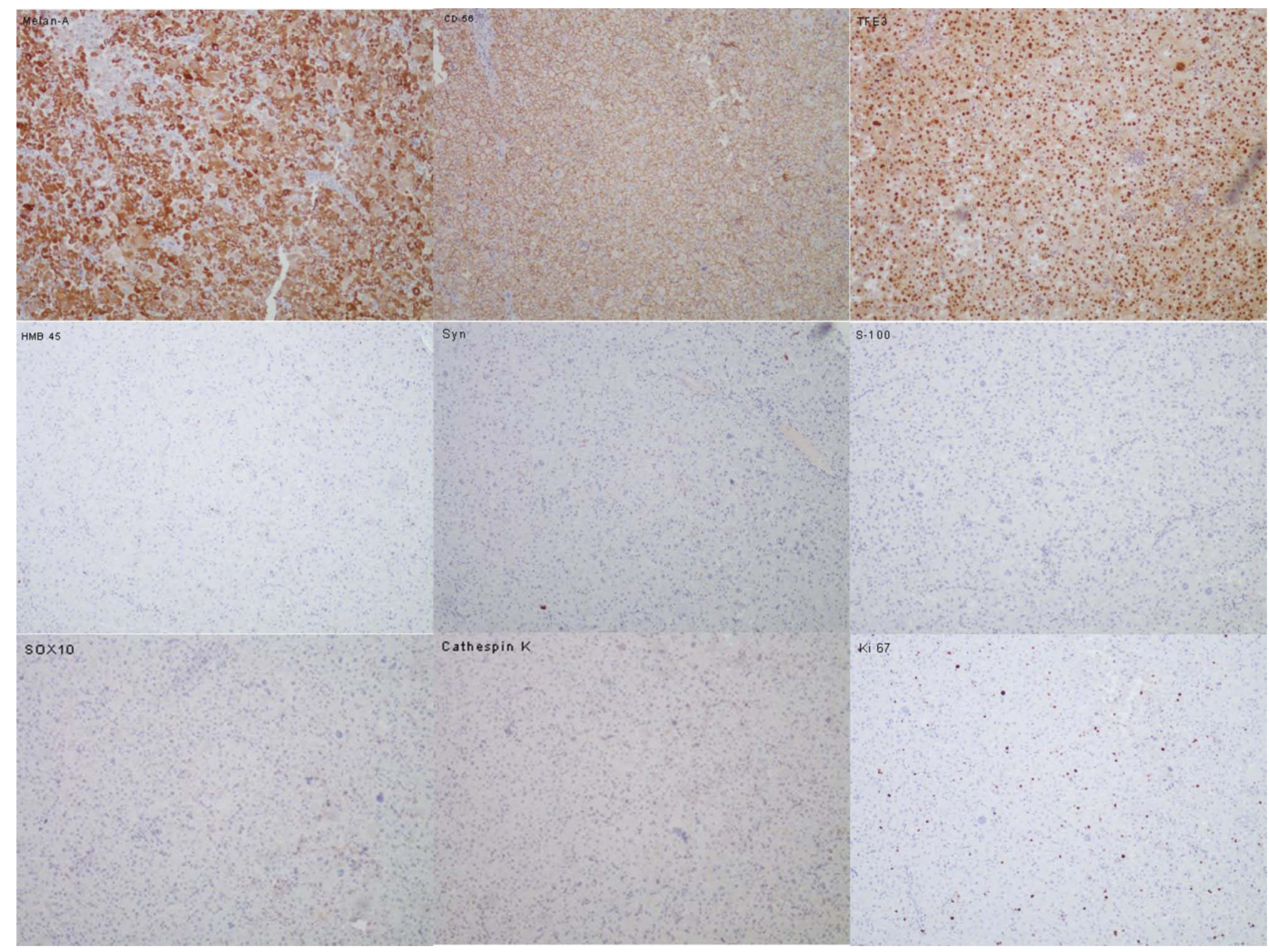

Figure 3 Immunohistochemistry of the perirenal mass showed positive for Melan-A, cluster differentiation (CD)56, and TFE3, while negative for HMB45, Syn, S-I00 and SOX10. Ki67 was present in approximately $1 \%$ of tumor cells. 
such as gastrointestinal tract, ${ }^{4}$ kidney,${ }^{12}$ pancreas, ${ }^{13}$ liver, ${ }^{14}$ ovary, ${ }^{15}$ uterus ${ }^{5}$ and so on. ${ }^{16,17}$ According to the recent systematic reviews, ${ }^{10,15}$ the most commonly affected organs were uterus (including uterine and cervix uteri) and colon, with approximately 65 and 20 cases reported online, respectively. Perirenal PEcoma are extremely rare, to date, only four cases have been reported and our case is the fifth one (Table 1). Besides, this case is the second case of perirenal PEComa complicated with other primary tumor. Demographically, perirenal PEComa mainly occurs in middle-aged and elderly women while our case involved a middle-aged man. No certain location preference and morphological features were found.

The PEcoma coexpresses melanocytic and muscle markers characteristically. Immunohistochemistry plays a vital role in the diagnosis. According to a systematic review in $2020,{ }^{18}$ HMB45, a specific marker for PEComa, abundantly clear to eosinophilic granular cytoplasm, is $99 \%$ positive in PEComa and has been utilized to support the diagnosis of PEComa at multiple sites. ${ }^{19}$ The positive rate of Melan-A, which is recognized as an antigen on melanoma cells by cytotoxic T-lymphocytes, varied from $13 \%$ to $88 \%$ in PEComa 18 . In addition, MTF, HMSA- $1,{ }^{20}$ smooth muscle actin (SMA), desmin ${ }^{21}$ and CathepsinK ${ }^{22}$ were successively reported to be potential markers in the diagnosis of the PEComa, yet no consensus has been reached. CD56 (+) supported the origins of neural tissue while S100 were usually negative in PEComa. ${ }^{23}$ Consistent with data shown in Table 1, immunoactivity for HMB45 was most commonly demonstrated, present in $4 / 5$ cases. Notably, our case showed positive expression for Melan-A while negative expression for HMB45 in perirenal lesion. Combining HMB45-negative cases occurring in other locations, ${ }^{10,23}$ more studies are still needed to reveal the certain pattern of the immunohistochemical features of PEComa.

In this case, the patient was admitted to the hospital due to pulmonary nodules considered as adenocarcinoma and the perirenal mass was found by further examination. Since kidney is one of metastasis sites of lung adenocarcinoma, and lung is a common metastatic organ of many malignant tumors, including primary malignant tumors in the kidney, we could not distinguish whether the perirenal neoplasm was a metastatic tumor or a primary tumor through image tests including enhanced CT and PET-CT. Finally, pathology of the pulmonary mass suggested MIA ( $<0.6 \mathrm{~cm}$ invasion), and immunohistochemistry stains suggested that the perirenal neoplasm was not the metastasis of pulmonary MIA but the primary PEComa. Similar to the other case, ${ }^{7}$ as most PEComa showno specific symptoms, the distinction between one primary tumor with metastatic sites and concomitantly primary tumors was difficult when imaging detected multiple lesions. On this occasion, the acquisition of specimens in multiple lesions is crucial for the diagnosis and the selection of the treatment. It is essential for pathologists to know the lesion to avoid misdiagnosis.

As a rare mesenchymal soft tissue neoplasm, no consensus has been reached on the treatment of PEComa. The classification and treatment of PEComa were significantly associated with six factors, including tumor size, infiltrative growth pattern, high nuclear grade cellularity, mitotic rate, necrosis and vascular invasion. ${ }^{3}$ However, considering the limited data, we could not conclude a similar classification targeted to the perirenal or retroperitoneal PEComa. In our case, the patient had no clinical symptoms and most of the other auxiliary examinations were normal. Besides, tumor size $(40 \times 20 \times 10 \mathrm{~mm})$ was consistent with the characteristics of benign tumor and no sign of progression was detected after the complete resection. Combined with the pathologic results, we confirmed that the perirenal PEComa was a benign lesion. Similarly, three other patients with perirenal PEComa with no obvious clinical symptoms, tumor size $\leq 10 \mathrm{~cm}$ and postoperative pathology suggesting no obvious nuclear heterogeneity, showed different degrees of improvement after the prompt surgery, which indicates that small tumor size, high nuclear grade and high cellularity are benign manifestations. Complete operation might be conducive to patients with perirenal PEComa that presents a benign phenotype, regardless of complications with other tumors. On the other hand, for patients with PEComa that shows malignant phenotype without resectability, both mTOR inhibitor and chemotherapy might not considerably improve the survival rate. ${ }^{7}$

\section{Conclusion}

To conclude, perirenal PEComa is a rare entity with no certain pathologic features and immunohistochemistry might play a crucial role in diagnosis. The acquisition of specimens in multiple lesions is vital to the distinction between one primary tumor with metastatic sites and concomitantly primary tumors. We hope that this case may contribute to an improved understanding of this disease and ultimately to avoid clinical misdiagnosis, missed diagnosis and treatment delay. Considering the limitations of the data, more studies are needed to provide new and comprehensive evidence for the diagnosis and treatment of PEComa. 


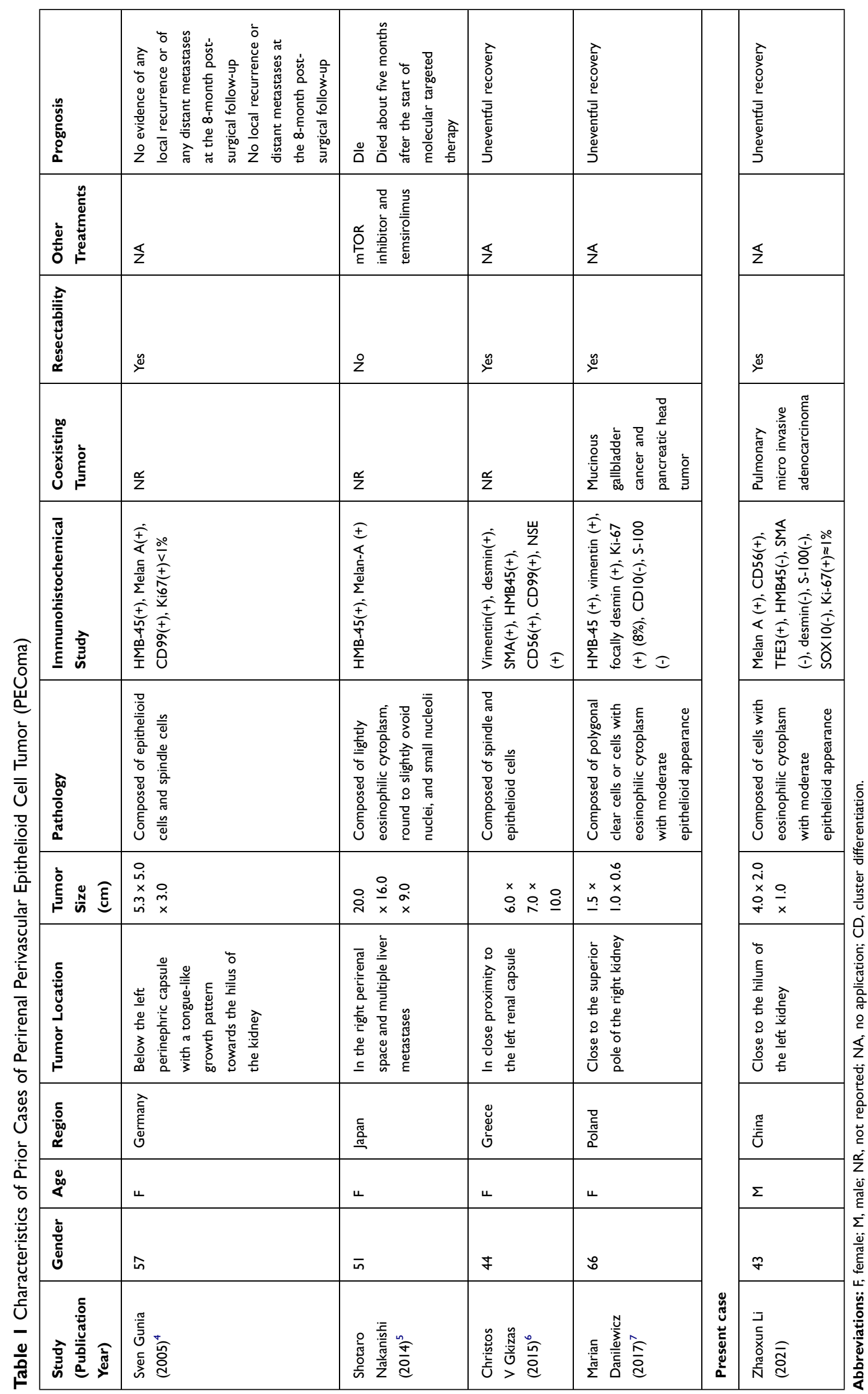




\section{Author Contributions}

All authors contributed to data analysis, drafting or revising the article, gave final approval of the version to be published, and agree to be accountable for all aspects of the work.

\section{Funding}

There is no funding to report.

\section{Disclosure}

The authors report no conflicts of interest in this work.

\section{References}

1. Zamboni G, Pea M, Martignoni G, et al. Clear cell "sugar" tumor of the pancreas. A novel member of the family of lesions characterized by the presence of perivascular epithelioid cells. Am J Surg Pathol. 1996;20(6):722. doi:10.1097/00000478-199606000-00010

2. Folpe AL, Cdm F, Unni KK, Epstein J, Mertens F. Pathology and Genetics of Tumours of Soft Tissue and Bone. IARC Press; 2002.

3. Folpe AL, Mentzel T, Lehr HA, Fisher C, Balzer BL, Weiss SW. Perivascular epithelioid cell neoplasms of soft tissue and gynecologic origin: a Clinicopathologic Study of 26 cases and review of the literature. Am J Surg Pathol. 2005;29(12):1558-1575. doi:10.1097/ 01.pas.0000173232.22117.37

4. Chen Z, Han S, Wu J, Xiong M, Song W. A systematic review: perivascular epithelioid cell tumor of gastrointestinal tract. Medicine. 2016;95(28):e3890. doi:10.1097/MD.0000000000003890

5. Musella A, De Felice F, Kyriacou AK. Perivascular epithelioid cell neoplasm (PEComa) of the uterus: a systematic review. Int J Surg. 2015;19:1-5. doi:10.1016/j.ijsu.2015.05.002

6. Danilewicz M, Strzelczyk JM, Wagrowska-Danilewicz M. Perirenal perivascular epithelioid cell tumor (PEComa) coexisting with other malignancies: a case report. Pol J Pathol. 2017;68:92-95.

7. Gkizas CV, Tsili AC, Katsios C, Argyropoulou MI. Perirenal PEComa: computed tomography findings and differential diagnosis. J Clin Imaging Sci. 2015;5(1):69. doi:10.4103/2156-7514.172977

8. Nakanishi S, Miyazato M, Kan Y, Tamashiro T, Saito S. [Perirenal malignant perivascular epithelioid cell tumor originating from right retroperitoneum: a case report]. Hinyokika Kiyo. 2014;60 (12):627-630. Japanese.

9. Gunia S, Awwadeh L, May M, Roedel S, Kaufmann O. Perivascular epithelioid cell tumor (PEComa) with perirenal manifestation. Int $J$ Urol. 2010;12(5):489-492. doi:10.1111/j.1442-2042.20 05.01077.x

10. Idania L, Ileana F, Olindo M, Roberta G, Paolo CR. Perivascular epithelioid cell tumour with intraorbital location: report of a case and review of the literature. Case Rep Pathol. 2016;2016:1936421.
11. Lantuejoul S, Rouquette I, Brambilla E, Travis WD. New WHO classification of lung adenocarcinoma and preneoplasia. Ann Pathol. 2016;36(1):5. doi:10.1016/j.annpat.2015.11.010

12. Yang Y, Yu X, Lu B, Shao Z. Perivascular epithelioid cell tumor (PEComa) of the kidney: an overview of its management and outcomes. J Int Med Res. 2020;48(10):030006052096122. doi: $10.1177 / 0300060520961223$

13. Zizzo M, Ugoletti L, Tumiati D, et al. Primary pancreatic perivascular epithelioid cell tumor (PEComa): a surgical enigma. A systematic review of the literature. Pancreatology. 2018;18(3):238-245.

14. Mitchell A, Jordan A, Lewit R, Kruse E. Perivascular epithelioid cell tumor of the liver. Am Surg. 2018;84(9):e428-e430. doi:10.1177/ 000313481808400933

15. Fitzpatrick M, Pulver T, Klein M, Murugan P, Khalifa M, Amin K. Perivascular epithelioid cell tumor of the uterus with ovarian involvement: a case report and review of the literature. Am J Case Rep. 2016;17:309-314. doi:10.12659/AJCR.896401

16. Zhong J, Hu Y, Si L, et al. Primary perivascular epithelioid cell tumor (PEComa) in bone: a review of the literature and a case arising in the humerus with multiple metastases. $J$ Bone Oncol. 2020;26. doi:10.1016/j.jbo.2020.100336. eCollection $2021 \mathrm{Feb}$.

17. Stuart LN, Tipton RG, Dewall M, et al. Primary cutaneous Perivascular Epithelioid Cell Tumor (PEComa): five new cases and review of the literature. J Cutan Pathol. 2017;44(8):713-721. doi:10.1111/cup. 12972

18. Bennett JA, Oliva E. Perivascular epithelioid cell tumors (PEComa) of the gynecologic tract. Genes Chromosomes Cancer. 2020;60 (3):168-179.

19. Bennett JA, Braga AC, Pinto A, Vijver K, Oliva E. Uterine PEComas: a morphologic, immunohistochemical, and molecular analysis of 32 tumors. Am J Surg Pathol. 2018;42(10):1. doi:10.1097/ PAS.0000000000001119

20. Schoolmeester JK, Howitt BE, Hirsch MS, Cin PD, Quade BJ, Nucci MR. Perivascular epithelioid cell neoplasm (PEComa) of the gynecologic tract: clinicopathologic and immunohistochemical characterization of 16 cases. Am J Surg Pathol. 2014;38(2):176-188. doi:10.1097/PAS.0000000000000133

21. Fang CL, Lin YH, Chen WY. Microscopic endometrial perivascular epithelioid cell nodules: a case report with the earliest presentation of a uterine perivascular epithelioid cell tumor. Diagn Pathol. 2012;7 (1):117. doi:10.1186/1746-1596-7-117

22. Rao Q, Cheng L, Xia Q-Y, et al. Cathepsin K expression in a wide spectrum of perivascular epithelioid cell neoplasms (PEComas): a clinicopathological study emphasizing extrarenal PEComas. Histopathology. 2013;62(4):642-650. doi:10.1111/his.12059

23. Ando K, Fujino N, Mitani K, et al. Isolation of individual cellular components from lung tissues of patients with lymphangioleiomyomatosis. Am J Physiol Lung Cell Mol Physiol. 2016;310(10):L899-L908. doi:10.1152/ajplung.00365.2015
OncoTargets and Therapy

\section{Publish your work in this journal}

OncoTargets and Therapy is an international, peer-reviewed, open access journal focusing on the pathological basis of all cancers, potential targets for therapy and treatment protocols employed to improve the management of cancer patients. The journal also focuses on the impact of management programs and new therapeutic agents and protocols on patient perspectives such as quality of life, adherence and satisfaction. The manuscript management system is completely online and includes a very quick and fair peer-review system, which is all easy to use. Visit http://www.dovepress.com/ testimonials.php to read real quotes from published authors. 